\title{
Simultaneous Impact of High Pressures and Pulsed Electric field on Saccharomyces cerevisiae Model System
}

\author{
Rzoska SJ',2*, Musiał $F^{1}$, Rutkowska ${ }^{1}$, Fonberg-Broczek $\mathbf{M}^{1}$, Sokołowska $B^{1,3}$, Drozd-Rzoska $A^{1}$ and Nowakowska $\mathrm{J}^{4}$ \\ ${ }^{1}$ Institute of High Pressure Physics, Polish Academy of Sciences, Warsaw, Poland \\ ${ }^{2}$ Silesian Centre for Interdisciplinary Education and Research, Institute of Physics, University of Silesia, Chorzów, Poland \\ ${ }^{3}$ Professor, Wacław Dabrowski Institute of Agricultural and Food Biotechnology, Departmentof Fruit and Vegetable Product Technology, Warsaw, Poland \\ ${ }^{4}$ Laboratory of Electron and Confocal Microscopy, Department of Biology, Warsaw University, Warsaw, Poland
}

\begin{abstract}
Results of simultaneous high pressure (HPP) and pulsed electric field (PEF) preservation treatments on Saccharomyces cerevisiae model suspension are presented. They are based on the novel implementation of PEF method, associated with the sequence of sine- wave pulses of the strong electric field. The simultaneous HPP + PEF enabled reaching the pasteurization threshold for pressure $\mathrm{P}=200 \mathrm{MPa}$ and the intensity of the electric field $\mathrm{E}<10 \mathrm{kV} / \mathrm{cm}$ well below typical values for separate HPP and PEF treatments. Notable is the negligible parasitic heating for the new PEF implementation. The supplementary broad band dielectric spectroscopy (BDS) frequency scan for HPP, PEF and HPP + PEF revealed qualitative differences, particularly notable for the complex conductivity representation.
\end{abstract}

Keywords: Non-thermal preservation of foods and biomaterials; High pressures; Strong electric field; Broad band dielectric spectroscopy

\section{Introduction}

Innovative, non-thermal methods for foods preservation have reached a significant position in the modern food science and technology. They aim at maintaining the quality of fresh products, avoiding notable deterioration of relevant properties during processing and storage [15]. This is hardly, if at all, possible for basic preservation methods, such as the thermal pasteurization, addition of chemical agents or radiation. Amongst innovative methods of foods preservation the high pressure preservation/processing (HPP) has reached the most notable market position. In its basic implementation pressures between $400 \mathrm{MPa}$ and $600 \mathrm{MPa}$, at near room temperatures, are exerted on already packed foods for few minutes time. For HPP the pasteurization threshold, i.e. 5 decades decrease of the total number of microorganisms, is most often linked to the pressure-induced break of cellular walls [1-8]. The technological sterilization target is reached for simultaneous actions of high pressure and temperature. This is known as the high pressure high temperature processing (HPHT). For this technology pressurization is carried out for temperatures $T \sim 120^{\circ} \mathrm{C}$, below the value typically used for sterilization under atmospheric pressure but well above the thermal pasteurization threshold $\left(\sim 80^{\circ} \mathrm{C}\right)$, associated with proteins' denaturation. Consequently, for HPHT method basic advantages of HPP technology are partially lost. Notwithstanding, HPHT technology also indicates possible advantages of the simultaneous, common action of two different physical factors $[2,4,8,9]$.

Increasing position within innovative non-thermal methods for foods preservation gains the application of pulsed electric fields (PEF), for which the strong electric field permeability of cellular is recognized as underlying phenomenon [10,11]. For this method different forms of pulses, described via electric field intensity vs. time dependences, are considered. However, in practice the discharge of energy collected in a set of capacitors is used. This can be associated with the necessity of the big energy output in a very short time.

The satisfactorily anti-microbial PEF action in foods is reached after a sequence of tens/hundreds of pulses, lasting at least tens of microseconds. Depending on the product, electric field intensity and the total duration of pulses the number of microorganisms can decrease from 2 to 5 decades [10-13]. For PEF method the post-process increase of temperature, reaching even $40 \mathrm{~K}$, is reported [12-14]. In the opinion of the authors the fact that the electric field act within a 'thin layer" in a very short time may indicate that the local heating can be even larger. It is notable that for PEF treatment some degree of denaturation and loss of microorganisms is reported, what may be considered as a possible aftermath of short-time local thermal pasteurization. The extensive resume of recent advances for HPP and PEF methods can be found in references [1-4,10-16]. This report presents studies focusing on the simultaneous impact of HPP + PEF on Saccharomyces cerevisiae model system. The behavior pre/post- HPP, PEF and HPP + PEF treatments was monitored via broad band dielectric spectroscopy (BDS) frequency scan. The research aimed on the preliminary answer to the question whether the hybrid, simultaneous action of strong electric fields and high pressures can yield any added value important for future implementations. Notable, are different physical origins of PEF and HPP antimicrobial action [14-16].

\section{Experimental}

The optimal electric 'pass through' into the interior of the pressure chamber should ensure high electric resistivity and the transfer of high voltage and high electric current signals. These should take place for hundreds of high pressure experiments. The 'pass through' element, developed in the Institute of High Pressure Physics Polish Academy

${ }^{*}$ Corresonding author: Institute of High Pressure Physics Polish Academy of Sciences, Warsaw, Poland, Tel: 0048-660-438-596; E-mail: sylwester.rzoska@ gmail.com

Received November 10, 2015; Accepted December 03, 2015; Published December 09, 2015

Citation: Rzoska SJ, Musiał F, Rutkowska, Fonberg-Broczek M, Sokołowska B et al. (2015) Simultaneous Impact of High Pressures and Pulsed Electric field on Saccharomyces cerevisiae Model System. J Food Process Technol 6: 518 doi:10.4172/2157-7110.1000518

Copyright: @ 2015 Rzoska SJ, et al. This is an open-access article distributed under the terms of the Creative Commons Attribution License, which permits unrestricted use, distribution, and reproduction in any medium, provided the original author and source are credited. 
Citation: Rzoska SJ, Musiał F, Rutkowska, Fonberg-Broczek M, Sokołowska B, et al. (2015) Simultaneous Impact of High Pressures and Pulsed Electric field on Saccharomyces cerevisiae Model System. J Food Process Technol 6: 518. doi:10.4172/2157-7110.1000518

Page 2 of 5

of Sciences (IHPP PAS) for nonlinear dielectric spectroscopy studies, is characterized by: resistivity $R \sim 1 G \Omega, I_{\max } \sim 0 A$ and $\Delta T<1 K[17-$ 20]. The latter is well below typical 5 voltage used in PEF applications for foods: $\mathrm{E}=30-100 \mathrm{kV} / \mathrm{cm}$ [14-16]. Such intensities for $\mathrm{U} \sim 1000 \mathrm{~V}$ can be reached only for the distance between electrodes $0.3 \mathrm{~mm}<\mathrm{d}$ $<0.1 \mathrm{~mm}$. However, this have to result in $(i)$ undesired very small resistivity and consequently (ii) extra parasitic heating, (iii) increasing probability of electric breakdowns, figure 1 shows the new PEF apparatus developed for studies which results are presented below. The desired PEF impact on microorganisms was reached for notably lower intensities of the electric field than used so far. This means avoiding undesirable factors listed above ((i), (ii), (iii)).

It bases on high voltage amplifiers (up to $2000 \mathrm{~V}$ peak-peak), with the high energy output reaching $500 \mathrm{~J}$. So far these parameter were obtained for the band up to $f=20 \mathrm{kHz}$. For higher frequency works are under development: max $500 \mathrm{~V}$ peak-peak was reached. It is notable that a similar design, although with lesser energy output, was used in dual field nonlinear dielectric effect (NDE) studies on super cooled liquids [17-20] and Electrooptic- Kerr Effect (EKE) investigations [21-24], also in water-based colloidal suspensions [22]. In these experiments changes of dielectric permittivity (NDE) or birefringence (EKE) vs. time are monitored. The undesired heating is detected on-line via the vertical shift of the baseline after strong electric field pulse action. However, these studies showed that the parasitic heating can be avoided if sine-wave pulses of high electric field with few $\mathrm{kHz}$ frequencies are applied [20,22]. This was explained by too large inertia of ionic species, responsible for heating, to be influenced by such form of the electric field. A similar effect was expected for Saccharomyces cerevisiae suspensions/colloids studies presented in this report in which the newPEF apparatus (Fig. 1) was used. As indicated below the implemented form of the strong electric field action decreased the voltage of the high electric to the level possible for application for the module with the sample placed within the high pressure chamber.
A notable feature of the design presented in figure 1 is also the flexibility in creating arbitrary forms and sequences of strong electric field pulses. For preliminary studies presented in this report, the strong electric field had the sine-wave form: each pulse contained ca. 10 periods of the electric field with $f=1,5$ and $10 \mathrm{kHz}$ frequencies. Tests were carried out at room temperature using the flat-parallel capacitor with diameter $d=40 \mathrm{~mm}$ and the distance up to $5 \mathrm{~mm}$, made from Invar. No impact of PEF action on capacitor plates was observed. This factor is worth stressing, since such impact is often reported in PEF studies carried out so far. The on-line digital oscilloscope monitoring of the action of the strong electric field in the newPEF apparatus was possible: no electric bids associated with the strong electric field were detected. The shift of the baseline prior and after the sequence of strong electric field pulses was negligible. The copper - constantan thermocouple located within one of plates showed that heating during the treatment was lesser than $\Delta T<1 K$. Immediately prior and after the newPEF treatment broad band dielectric spectroscopy (BDS) scans were carried out. In high pressure studies of liquid and soft matter samples under pressure the total isolation from the impact of the pressure transmitting liquid (mixture of octane and silicone oil in our cases) constitute the key experimental problem [25-27]. figure 2 shows the module designed and build for given studies, avoiding such undesired impact. It enabled BDS monitoring immediately prior and after HPP, PEF and HPP+PEF. BDS scan was carried out using Alpha Novo control analyzer, between $100 \mathrm{~Hz}$ and $10 \mathrm{MHz}$, which enabled permanent 5 digits resolution.

Suspensions of Saccharomyces cerevisiae NCFB 3191 strain were used. Twenty-four hour culture in YPG-broth ( $1 \%$ yeast extract-Difco, $1 \%$ peptone-Difco, $2 \%$ glucose-POCh) was centrifuged at $4^{\circ} \mathrm{C}$ for 10 min at $6000 \times \mathrm{g}$, and sedimented cells were aseptically re-suspended in phosphate-buffered saline (PBS, pH 7.2) and again centrifuged. The washing procedure was repeated twice more. The final suspensions

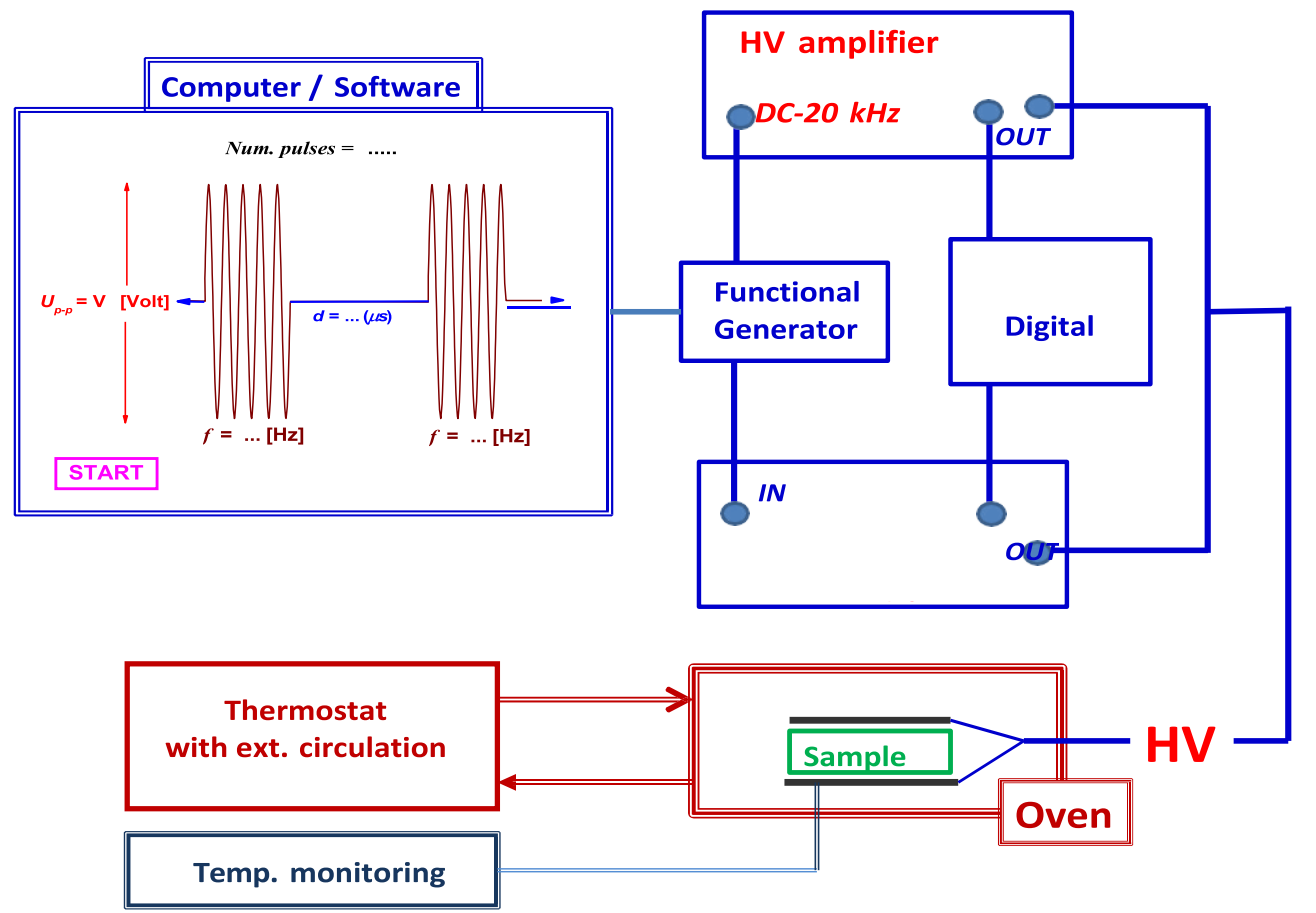

Figure 1: The scheme for Unipress experimental facility for pulsed electric field preservation/treatment (PEF) of foods and biomaterials 
Citation: Rzoska SJ, Musiał F, Rutkowska, Fonberg-Broczek M, Sokołowska B, et al. (2015) Simultaneous Impact of High Pressures and Pulsed Electric field on Saccharomyces cerevisiae Model System. J Food Process Technol 6: 518. doi:10.4172/2157-7110.1000518

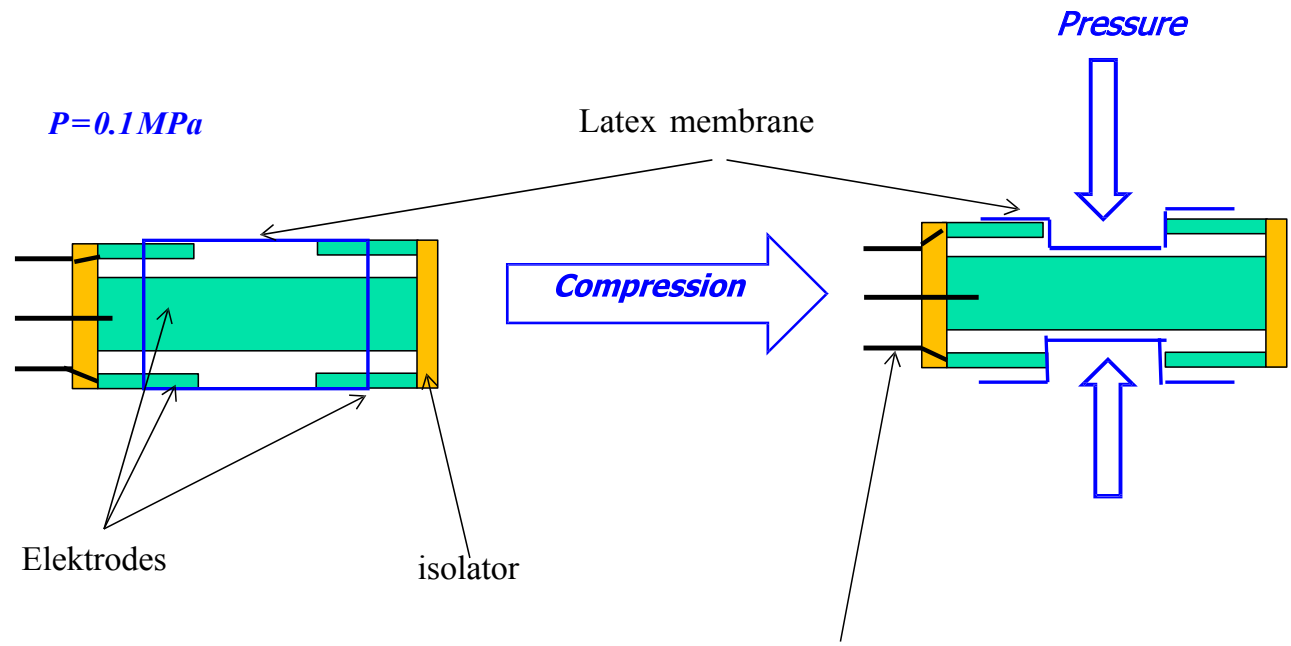

To the ,head" of the pressure chamber

Figure 2: The capacitor for the simultaneous HPP and PEF treatment, enabling immediate subsequent BDS monitoring.

of S. Cerevisiae were prepared in PBS, reaching cell concentration of about $10^{17}-10^{18} \mathrm{cfu} / \mathrm{g}$ (colony forming units/g, i.e. living cells/g) and in agarose gel, with the concentration of about $\left.10^{7}-10^{8} \mathrm{cfu} / \mathrm{g}\right)$. The suspension was prepared in water and in gel (agar) to reduce electric conductivity. Samples were centrifuged for $10 \mathrm{~min}$ at $3500 \times \mathrm{g}$ and prepared for the electron microscopy. The cells were fixed with $2.5 \%$ glutaraldehyde cacodylic buffer and incubated overnight, then washed in $0.1 \mathrm{M}$ cacodylic buffer ( $\mathrm{pH} 7.2$ ). Next the material was post-fixed in $1 \% \mathrm{OsO}_{4}$ in $\mathrm{ddH}_{2} \mathrm{O}$ for $3 \mathrm{~h}$ and washed three times in $\mathrm{ddH}_{2} \mathrm{O}$. After post-fixation, samples were dehydrated through a graded series of EtOH (50\% - $10 \mathrm{~min}, 70 \%$ - $24 \mathrm{~h}, 90 \%-10 \mathrm{~min}, 96 \%-10 \mathrm{~min}$,) and dried in the open air. Next, dry samples were mounted on aluminum stubs in different positions and coated with gold in sputter coater (POLARON SC7620) and were examined in LEO 1430VP scanning electron microscope produced by Zeiss(Germany).

\section{Results and Conclusions}

Results of newPEF action on Saccharomyces cerevisiae suspension under atmospheric pressure are shown in figure 3. It is related to following conditions: $\mathrm{E}=20 \mathrm{kV} / \mathrm{cm}$ with, 50 sine-wave pulses $(f=5$ $\mathrm{kHz}, 10$ cycles each) and 1 second delay between pulses it is possible to crossover the pasteurization threshold. The photo shows the strong destruction of intracellular organelles and the visible impact on the cell wall along with the reduction of living yeast cell number from $5.6 \times 10^{7}$ to $3.5 \times 10^{2}$, i.e. 5.2 decade (hence the pasteurization threshold - 5 decades - was reached). The increase of temperature was only $0.5 \mathrm{~K}$ during processing. For other tested frequencies at one decade smaller decrease of the number of microorganisms was noted. Results of the hybrid, simultaneous actions of HPP and PEF are shown in figure 4. They are related to following condition $E=5 \mathrm{kV} / \mathrm{cm}, f=5 \mathrm{kHz}, 10$ cycles in each pulse with 15 pulses and $1 \mathrm{~s}$ distance between them for PEF and $P=200$ $\mathrm{MPa}$ and $t=5 \mathrm{~min}$ for HPP. Under such mild $(P, E)$ separate PEF or HPP can yield only a sub-lethal effect, with microorganism revitalizing after treatment. The hybrid PEF+HPP treatment resulted in $5.8 \mathrm{log}$ units' reduction of the number of living cells. figure 5 Presents results of BDS scans of tested yeast samples: (i) native (prior to HPP/PEF treatments), (ii) after HPP treatment, (iii) after PEF action and (iv)after simultaneous $\mathrm{PEF}+\mathrm{HPP}$, treatments. BDS experimental results are

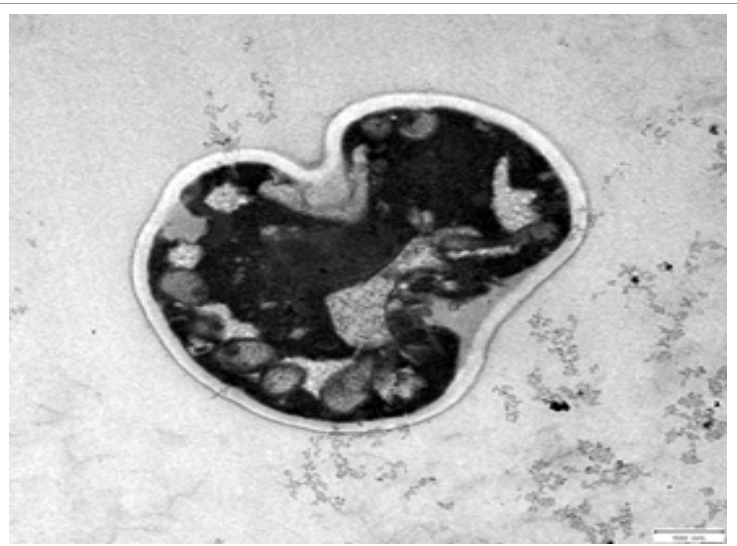

Figure 3: TEM picture of Saccharomyces cerevisiae cell after PEF treatment, basing on apparatus from Figure 1. Conditions: $E=20 \mathrm{kV} / \mathrm{cm}$ with, 50 sine-wave pulses ( $f=5 \mathrm{kHz}, 10$ cycles each) and 1 second delay between pulses.

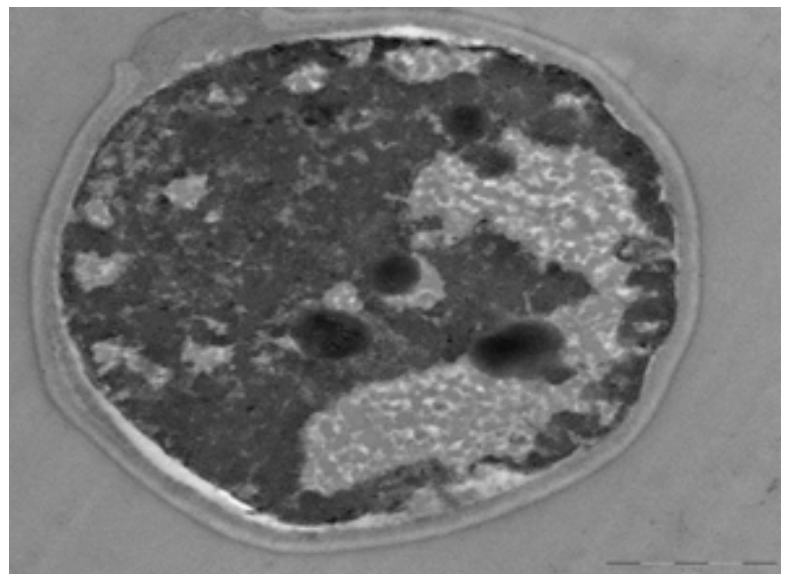

Figure 4: TEM picture of Saccharomyces cerevisiae cell after PEF treatment. Conditions: $E=5 \mathrm{kV} / \mathrm{cm}, \mathrm{f}=5 \mathrm{kHz}, 10$ cycles in each pulse with 15 pulses and $1 \mathrm{~s}$ distance between them for PEF and $\mathrm{P}=200 \mathrm{MPa}$ and $\mathrm{t}=5 \mathrm{~min}$ for HPP. 
Citation: Rzoska SJ, Musiał F, Rutkowska, Fonberg-Broczek M, Sokołowska B, et al. (2015) Simultaneous Impact of High Pressures and Pulsed Electric field on Saccharomyces cerevisiae Model System. J Food Process Technol 6: 518. doi:10.4172/2157-7110.1000518

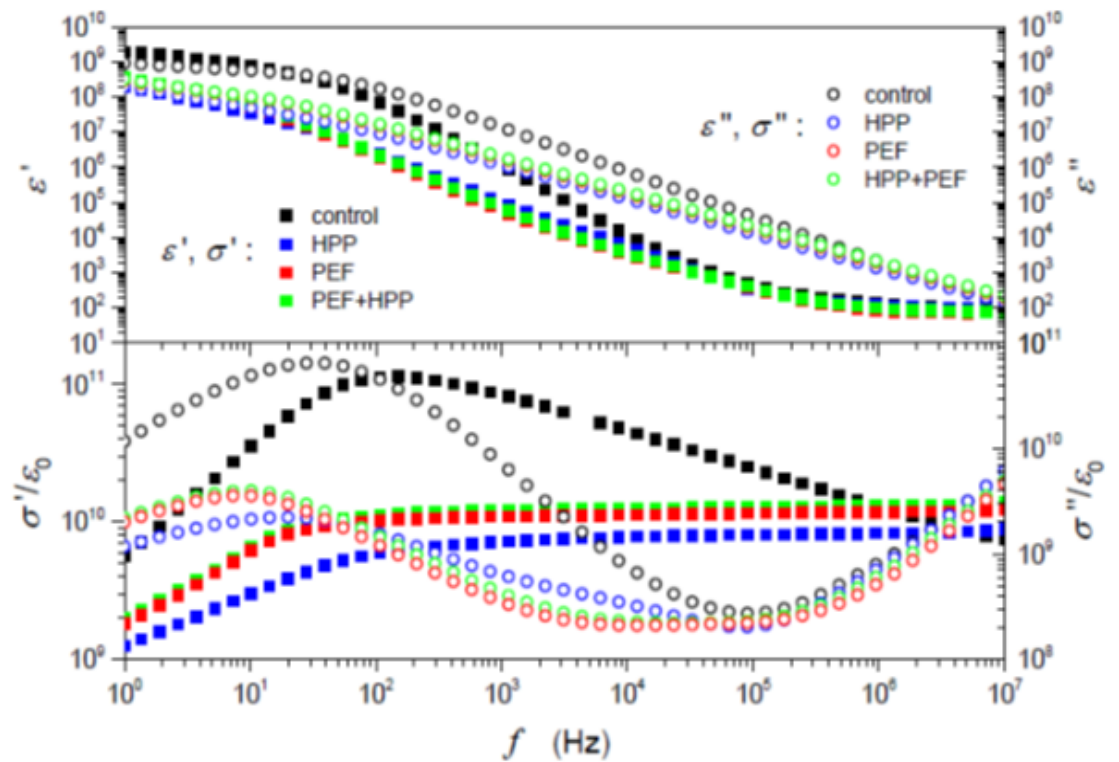

Figure 5: The BDS scan of dielectric properties for the basic yeast colloid/suspension and the same system after PEF, HPP and hybrid PEF + HPP treatments as discussed above. The upper part is for the complex dielectric permittivity and the lower one for the complex electric conductivity representation.

presented in two representations. First, in most common the complex dielectric permittivity representation [28].

$$
\varepsilon *(f)=\varepsilon^{\prime}(f)-i \varepsilon^{\prime \prime}(f)=\frac{C}{C_{0}}-\frac{i G}{\omega C_{0}}
$$

Where $C$ and $G$ are for capacitance and conductance, $C_{0}$ is for the empty capacitor and

$\omega=2 \pi f$ is for the angular frequency. However for highlighting features appearing in highly conductive media electric conductivity representation is often advised $[28,29]$ :

$$
\sigma *(f)=i \varepsilon_{0} \omega \varepsilon *=i \varepsilon_{0} \omega\left(\varepsilon^{\prime}-i \varepsilon^{\prime \prime}\right)=\varepsilon_{0} \omega \varepsilon^{\prime \prime}+i \varepsilon_{0} \omega \varepsilon^{\prime \prime}=\sigma^{\prime}+i \sigma^{\prime \prime}
$$

Jonsher $[28,30]$ showed that the real part of electric conductivity, often recalled simply as the conductivity, can be splitted into two terms: $\sigma=\sigma^{\prime}(f)=\varepsilon_{0} \omega \varepsilon^{\prime \prime}=\sigma_{D C}+\sigma_{A C} \approx \sigma_{D C}+A \omega^{S}$. The component $\sigma_{D C}$ is for the 'static', i.e. the frequency independent component of electric conductivity.

Regarding the behavior of $\varepsilon^{\prime \prime}(f)$, it first increases following the dependence $\varepsilon^{\prime \prime}(f) \propto f^{-k}$. For HPP, new PEF and HPP + newPEF treated samples the behavior is related to $k \approx 1$, what correlates with the constant $\sigma^{\prime \prime}(f)=\sigma_{D C}$ values from $10 \mathrm{MHz}$ to ca. $100 \mathrm{~Hz}$ in the electric conductivity representation (the lower part of figure 2). Such behavior is observed also in 'classical' fluids. It is associated with the fact that the constant number of ionic species can be gradually more 'slowly' moved by the electric field when decreasing its frequency. However, in the same range of frequencies for the native 'vivid' sample of yeast the coefficient $k \approx 1.25$, what is reflected by the linear increase of $\sigma^{\prime}(f)$ in the $\log$ - $\log$ scale $\sigma^{\prime}(f) \infty f^{1-k} \approx f^{-0.25}$. To the best of the authors knowledge this is the first ever evidence of such behavior in BDS studies in fluid samples. It may suggest that on decreasing frequency of the electric field the number of interacting with the field ionic species increases (!). On reaching the threshold frequency, equal to ca. $100 \mathrm{~Hz}$ for the control sample and $10-20 \mathrm{~Hz}$ for samples after 'cold pasteurizations", the exponent notably decreases and $k<1$.This can be explained as the results of the final polarization of capacitors plates via the Maxwell-Wegner effect [28]. The evolution of the real part of dielectric permittivity $\varepsilon^{\prime}(f)$ and the imaginary part of the conductivity $\sigma^{\prime \prime}(f)=\varepsilon_{0} \omega \varepsilon^{\prime}(f)$ show changes of polarization ability of ionic species. It is notable that for $f \rightarrow 10 \mathrm{MHz}$ the permittivity $\varepsilon^{\prime} \rightarrow 80$, i.e. the value characteristic for dielectric constant of water [28].

Concluding, results presented show that new developments for PEF treatments for foods and biomaterial make it possible to reduce qualitatively the parasitic heating. The simultaneous action of high pressures and strong electric fields (HPP + newPEF) caused the pasteurization threshold can be reached for notable lower pressures and weaker electric field than for separate HPP and PEF treatments. This can indicate an interesting perspective for the development of innovative, non-thermal methods for preservation of foods, pharmaceuticals, etc. Finally, it has been noted that the BDS frequency scan can serve as the convenient tool for on-line tests of features and 'quality' estimation of foods or biomaterials after HHP or PEF treatments.

\section{Acknowledgments}

This research was supported by National Centre for Science (NCN, Poland) via grant 2011/01/B/NZ9/02537.

\section{References}

1. Gutiérrez-Lopez GF, Barbosa-Cánovas GV, Welti-Chanes J, Parada-Arias E (2014) Food Engineering: Integrated Approaches. Berlin, Springer.

2. Jaeger H, Reineke K, Schoessler K, Knorr D (2012) Effects of emerging processing technologies on food material properties. Food Materials Science and Engineering, Wiley-Blackwell, Oxford. 
Citation: Rzoska SJ, Musiał F, Rutkowska, Fonberg-Broczek M, Sokołowska B, et al. (2015) Simultaneous Impact of High Pressures and Pulsed Electric field on Saccharomyces cerevisiae Model System. J Food Process Technol 6: 518. doi:10.4172/2157-7110.1000518

Page 5 of 5

3. Knorr D, Froehling A, Jaeger H, Reineke K, Schlueter O, et al. (2011) Emerging technologies in food processing. Ann Rev Food Sci and Technol 2: 203-235.

4. 4. Koutchma T (2014) Adapting High Hydrostatic Pressure (HPP) for Food Processing Operations. Amsterdam, Elsevier.

5. Sokołowska B, Skąpska S, Fonberg-Broczek M, Niezgoda J, Rutkowska M, et al. (2013) The influence of high pressure on survival of Saccharomyces cerevisiae in the model suspension and in beetroot juice. High Pressure Research 33: 165-171.

6. Sokołowska B, Skąpska S, Niezgoda J, Rutkowska M, Dekowska A, et al. (2014) Inactivation and sublethal injury of Escherichia Coli and Lysteria Innocua by high hydrostatic pressure in model suspensions and beetroot juice. High Pressure Research 34: 147-155.

7. Fonberg-Broczek M, Windyga B, Szczawiński J, Szczawińska M, Pietrzak D, et al. (2005) High pressure processing for food safety. Acta Biochim Pol 52: $721-723$

8. Vleeschouwer K, Van der Plancken L, Van Loey A, Hendrickx ME (2010) The Effect of High Pressure - High Temperature Processing Conditions on Acrylamide Formation and Other Maillard Reaction Compounds. J. Agric Food Chem 58: 11740-11748.

9. Matser AM, Krebbers B, Berg RW, Bartels PV (2004) Advantages of high pressure sterilization on quality of food products. Trends in Food Sci \& Technol 15: 79-85

10. Singh R, Kumar A (2011) Pulsed Electric Fields Processing and Application in Food Industry. Europ J Food Res \& Rev 1: 71-93.

11. Toepfl S (2011) Pulsed Electric Field food treatment - scale up from lab to industrial scale. Proc Food Sci 1: 776-779.

12. Wiktor A, Iwaniuk M, Śledź M, Nowacka M, Chudoba T, et al. (2013) Drying Kinetics of Apple Tissue Treated by Pulsed Electric Field. Drying Technol 31: 112-119.

13. Reineke K, Schottroff F, Meneses N, Knorr D (2015) Sterilization of liquid foods by pulsed electric fields-an innovative ultra-high temperature process. Front Microbiol 6: 400-410.

14. Jayaram SH (2013) Effect of pulse width on medium temperature rise and microbial inactivation under pulsed electric field food treatment. IEEE Trans on Indust Appl 49: 1767-1772.

15. Lelieveld HLM, Notermans S, De Haan (2007) Food Preservation by Pulsed Electric Fields. Springer, Berlin.

16. Stoica M, Bahrim G, Cârâc G (2011) Factors that Influence the Electric
Field Effects on Fungal Cells: Science against microbial pathogens: Communicating current research and technological advances. Formatex Research Centre, London.

17. Rzoska SJ, Zioło J, Drozd-Rzoska A (1997) Stretched-relaxation afte switching-off a strong electric field in a critical solution under high pressure. Phys Rev E 56: 2578-2581.

18. Rzoska SJ, Zioło J (1999) Dynamics of glassy clusters appearing by nonlinear dielectric effect studies, Phys Rev E 59: 2460-2465.

19. Drozd-Rzoska A, Rzoska SJ Zioło J (2008) Anomalous temperature behavior of nonlinear dielectric effect in supercooled nitrobenzene.

20. Rzoska SJ, Drozd-Rzoska A (2011) Dual field nonlinear dielectric spectroscopy in a glass forming EPON 828 epoxy resin. J Phys Condens Matter.

21. Mantegazza F, Giardini ME, Degiorgio V, Asnaghi D, Giglio M, et al. (1995) Electric birefringence study of reaction-limited colloidal aggregation. J Colloids and Interface Sci 170: 50-56.

22. Delgado AV, Carrique F, Arroyo FJ, Bellini T, Mantegazza F, et al. (1998) Frequency-dependence of the dielectric and electrooptic response in suspensions of charged rod-like colloidal particles. Colloids and Surface A Physicochem and Engn Aspects 140: 157-168.

23. Rzoska SJ (1993) Kerr effect and nonlinear dielectric effect on approaching the critical consolute point. Phys Rev 48: 1136-1143.

24. Rzoska SJ, Degiorgio V, Bellini T, Piazza R (1994) Relaxation of the electric birefringence near a critical consolute point. Phys Rev E 49: 3093-3096.

25. Drozd-Rzoska A, Rzoska SJ, Zioło J (1997) High pressure studies of the low-frequency nonlinear dielectric effect in the isotropic phase of octyl- and dodecyl- cyanobiphenyls. Phys Rev E 55: 2888-2891.

26. Rzoska SJ, Mukherjee PK, Rutkowska M (2012) Does the characteristic value of the discontinuity of the isotropic-mesophase transition in n-cyanobipheny exist? J Phys Condens Matt.

27. Floudas G, Paluch M, Grzybowski A, Ngai KL (2010) Molecular Dynamics of Glass-Forming Systems-Effect of Pressure. Springer-Verlag, Berlin

28. Kremer F, Schoenhals A (2003) Broadband dielectric spectroscopy. Springer Verlag, Berlin.

29. Ito N, Huang W, Richert R (2006) Dynamics of a Supercooled lonic Liquid Studied by Optical and Dielectric Spectroscopy. J Phys Chem 110: 4371-4377.

30. Jonsher AK (1983) Dielectric Relaxation in Solids. Chelsea Dielectric Press, London. 